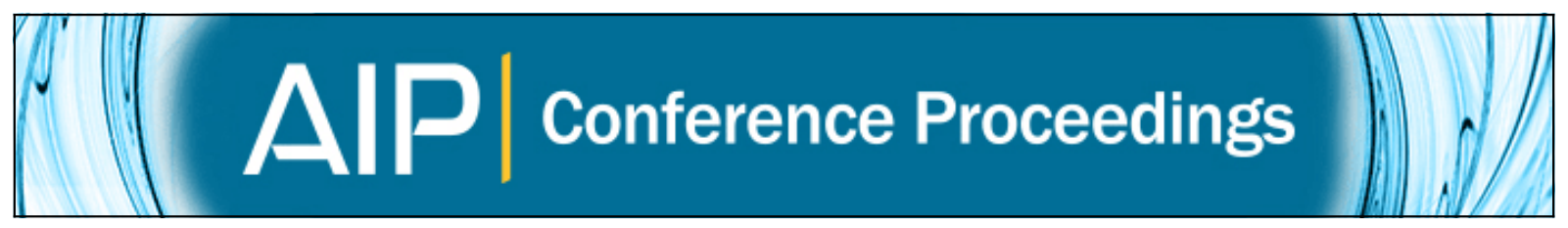

\title{
Ultra-sensitive transition-edge sensors (TESs) for far-IR/submm space-borne spectroscopy
}

M. Kenyon, P. K. Day, C. M. Bradford, J. J. Bock, and H. G. Leduc

Citation: AIP Conference Proceedings 1185, 56 (2009); doi: 10.1063/1.3292406

View online: http://dx.doi.org/10.1063/1.3292406

View Table of Contents: http://scitation.aip.org/content/aip/proceeding/aipcp/1185?ver=pdfcov

Published by the AIP Publishing

\section{Articles you may be interested in}

Optical performance of an ultra-sensitive horn-coupled transition-edge-sensor bolometer with hemispherical backshort in the far infrared

Rev. Sci. Instrum. 87, 043103 (2016); 10.1063/1.4945302

Fine pitch transition-edge sensor $\mathrm{X}$-ray microcalorimeters with sub-eV energy resolution at $1.5 \mathrm{keV}$

Appl. Phys. Lett. 107, 223503 (2015); 10.1063/1.4936793

Complex impedance, responsivity and noise of transition-edge sensors: Analytical solutions for two- and threeblock thermal models

AIP Advances 2, 042110 (2012); 10.1063/1.4759111

Normal metal-superconductor decoupling as a source of thermal fluctuation noise in transition-edge sensors J. Appl. Phys. 112, 034515 (2012); 10.1063/1.4745908

A cryogenic platform for space-borne instruments with nanoKelvin stability

AIP Conf. Proc. 613, 1249 (2002); 10.1063/1.1472152 


\title{
Ultra-sensitive transition-edge sensors (TESs) for far-IR/submm space-borne spectroscopy
}

\author{
M. Kenyon, P. K. Day, C. M. Bradford, J. J. Bock and H. G. Leduc
}

Jet Propulsion Laboratory (JPL), Pasadena,CA 91109

\begin{abstract}
We have built surface micromachined thin-film metallized $\mathrm{Si}_{x} \mathrm{~N}_{y}$ optical absorbers for transition-edge sensors (TESs) suitable for the Background-Limited far-IR/Submm Spectrograph (BLISS). BLISS is a broadband (38 $\mu \mathrm{m}-433 \mu \mathrm{m})$, grating spectrometer consisting of five wavebands each with a modest resolution of $\mathrm{R} \sim 1000$. Because BLISS requires the effective noise equivalent power (NEP) of the TES to be below $10^{-19} \mathrm{~W} / \mathrm{Hz}^{1 / 2}$, our TESs consist of four long $(1000 \mu \mathrm{m})$, narrow $(0.4 \mu \mathrm{m})$, and thin $(0.25 \mu \mathrm{m}) \mathrm{Si}_{x} \mathrm{~N}_{y}$ support beams that reduce the thermal conductance $G$ between the substrate and the optical absorber. To reduce the heat capacity of the absorber and make the response time $\tau$ fast enough for BLISS, our absorbers are patterned into a mesh geometry with a fill factor of less than $10 \%$. We use a bilayer of $\mathrm{Ti} / \mathrm{Au}$ to make the effective impedance of the absorber match the impedance of the incoming radiation for each band. Measurements of the response time of the metallized absorbers to heat pulses show that their heat capacity exceeds the predictions of the Debye model. Our results are suggestive that the surface of the low pressure chemical vapor deposition (LPCVD) $\mathrm{Si}_{x} \mathrm{~N}_{y}$ used in the absorbers' construction is the source of microstates that dominate the heat capacity.
\end{abstract}

Keywords: transition-edge sensor; far-IR spectrometer; submillimeter spectrometer; $\mathrm{Si}_{x} \mathrm{~N}_{y}$ heat capacity PACS: $85.25 . \mathrm{Pb} ; 95.85 . \mathrm{Gn} ; 95.85 . \mathrm{Fm} ; 63.22 .+\mathrm{m}$

\section{INTRODUCTION}

The Background-Limited Far-IR/Submm Spectrograph (BLISS) is a grating spectrometer with a resolving power of $\mathrm{R} \sim 1000$ and waveband coverage from $38 \mu \mathrm{m}-$ $433 \mu \mathrm{m}$ [1]. It has been funded for concept study by a broad team of US scientists led by the Jet Propulsion Laboratory. On a large aperture space telescope cooled to $5 \mathrm{~K}$, BLISS will radically advance our knowledge of the far-IR/submm universe and address science themes such as the history of star and galaxy formation and black hole growth [2].

This pioneering instrument could be launched late next decade aboard SPICA, a 3-meter class cryogenic far-IR observatory under study by the Japanese and European astrophysics communities and envisioned for a 2017 launch [3]. Success on SPICA would pave the way for an even more capable spectrometer for SAFIR/CALISTO-a large, $5 \mathrm{~K}$ observatory to be deployed at L2 in the 2020 decade. BLISS for SPICA and the future missions requires the development of a large format focal plane architecture with a sensitivity approaching the natural astrophysical backgrounds. Because BLISS is a spectrometer, the sensitivity of detectors for BLISS requires a noise-equivalent power (NEP) less than $1 \times 10^{-19} \mathrm{~W} / \mathrm{Hz}^{1 / 2}$

We have previously shown that the NEP of membraneisolated transition-edge superconducting bolometers (TESs) can be made very low $[4,5,6]$. In particular, we have built TESs consisting of a superconducting thermistor patterned onto a suspended, thermally isolated absorber that is connected to the substrate through four $\mathrm{Si}_{x} \mathrm{~N}_{y}$ support beams. By making the aspect ratio of the length-to-cross-sectional area of the support beams very large, we have shown that the thermal conductance $G$ between the absorber and substrate can be lowered far below $100 \mathrm{fW} / \mathrm{K}$. If the TES is operated below $100 \mathrm{mK}$, then the fundamental intrinsic temperature-fluctuation noise due to phonon fluctuations between the absorber of the bolometer and the substrate can be lowered below $1 \times 10^{-19} \mathrm{~W} / \mathrm{Hz}^{1 / 2}$.

In addition to a low NEP, BLISS requires that the response time $\tau$ of the TES must be below about $100 \mathrm{~ms}$. The response time $\tau$ is proportional to $C / G$ where $C$ is the heat capacity of the absorber and $G$ is the thermal conductance of the support beams. Because the thermal conductance $G$ is so small for TESs suitable for BLISS, it is necessary to reduce the heat capacity $C$ below about $100 \mathrm{fJ} / \mathrm{K}$. (We assume electrothermal feedback increases the response time by a factor of 10-100 [7].) For the longer wavelength spectrograph modules for BLISS, this becomes a challenge since it is necessary to make absorbers as large as $2.1 \mathrm{~mm} \times 336 \mu \mathrm{m}$ (see Table I).

Here we present our results of a detailed study of our optical absorbers designed for the longer wavelength bands. As in our previous work [8], we find that the heat capacity is larger than the predictions of the Debye model, behaving instead more like a glassy material at low temperature [9]. This behavior has been seen by others $[10,11]$ in amorphous and non-stoichiometric low pressure chemical vapor deposition $\mathrm{Si}_{x} \mathrm{~N}_{y}$. Because of the constraint that $\tau \simeq 100 \mathrm{~ms}$, this high specific heat 
TABLE 1. Spectral bands for BLISS. Bands 1 and 2 use a cross-dispersed echelle grating requiring quasi2D arrays and bands 3-5 use a planar waveguide grating demonstrated in the ground-based prototype Z-Spec [12], requiring $1 \mathrm{D}$ arrays. For bands $3-5, \Delta \mathrm{x}$ is the separation of the two plates that define the waveguide and $\Delta y$ is the pitch of the 1D arrays which are mounted along the edge of the waveguide [1].

\begin{tabular}{ccccc}
\hline \hline Band & $\lambda_{\text {min }}(\mu \mathrm{m})$ & $\lambda_{\text {max }}(\mu \mathrm{m})$ & Pixel Size: $\Delta \times(\mu \mathrm{m})$ & Pixel Size: $\Delta y(\mu \mathrm{m})$ \\
1 & 38 & 67 & 306 & 193 \\
2 & 67 & 116 & 535 & 337 \\
3 & 116 & 180 & 873 & 140 \\
4 & 180 & 280 & 1353 & 216 \\
5 & 280 & 433 & 2097 & 336 \\
\hline \hline
\end{tabular}

may limit whether we can build TESs with a $G$ small enough to lower the NEP to the background limit of $3 \times$ $10^{-20} \mathrm{~W} / \mathrm{Hz}^{1 / 2}$.

\section{FABRICATION}

Figure 1(a) shows a micrograph of a segment of a 1D absorber-coupled TES array which was fabricated at the Microdevices Laboratory (MDL) at JPL from bare 100 $\mathrm{mm} \mathrm{Si}$ wafers to finished arrays. (The optical design of BLISS requires 1D arrays.) The thermistors are composed of sputtered Mo/Au bilayer films that are lithographically patterned onto a suspended, thermally isolated low-pressure chemical vapor deposition (LPCVD) $\mathrm{Si}_{x} \mathrm{~N}_{y}$ absorber with a thickness of $0.25 \mu \mathrm{m}$. The stress of the $\mathrm{Si}_{x} \mathrm{~N}_{y}$ is carefully controlled to produce absorbers that are flat to within a few microns. Each absorber is $\Delta \mathrm{y}=300 \mu \mathrm{m}$ wide by $\Delta \mathrm{x}=2000 \mu \mathrm{m}$ long and designed to mount into the longest-wavelength band of BLISS (see Table 1 for details). The impedance of the absorber is matched to the incident radiation by depositing a very thin Au film on top of the absorber (see ref. [8] for more details). The incident radiation is polarized and perpendicular to the support beams.

We tune the transition temperature $T_{c}$ (roughly the operating temperature) of the TES by adjusting the thickness of the Mo and Au films. Four support beams connect the absorber to the substrate. (These support beams extend beyond the edge of the micrograph.) The geometry of the support beams for the TESs are $1000 \mu \mathrm{m}$ long by $0.25 \mu \mathrm{m}$ thick by $0.4 \mu \mathrm{m}$ wide. Sputtered aluminum lines run over the support beams and electrically connect the thermistor to a bias and readout circuit. Note the high aspect ratio of length-to-cross-sectional area of the support beams. The key to making this technology suitable for BLISS is to lower G by making support beams with a very high length-to-cross-sectional area aspect ratio. This high ratio is necessary to lower the thermal conductance between the absorber and the substrate.

Below $116 \mu \mathrm{m}$ (Bands 1 and 2), we need to add bends

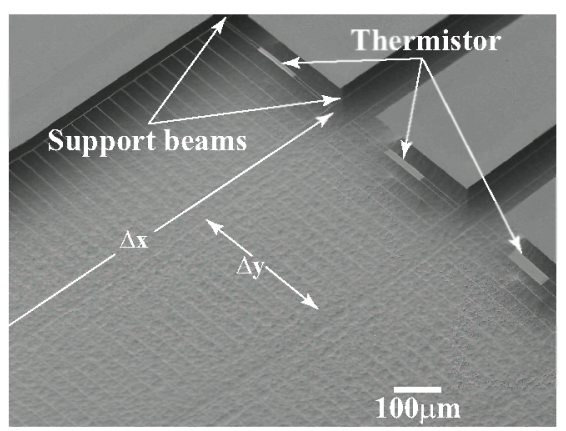

FIGURE 1. Micrograph of a segment of a 1D array of TESs with suspended $\mathrm{Si}_{x} \mathrm{~N}_{y}$ absorbers. Each absorber is $\Delta y=300$ $\mu \mathrm{m}$ wide by $\Delta \mathrm{x}=2000 \mu \mathrm{m}$ long (see Table 1 for details) and designed to mount into the longest-wavelength band of BLISS. The beam dimensions are $1000 \mu \mathrm{m}$ long by $0.25 \mu \mathrm{m}$ thick by $0.4 \mu \mathrm{m}$ wide. (Note: the beams extend beyond the edge of the micrograph.) The absorber consists of parallel wires with a width $w=0.5 \mu \mathrm{m}$ and spacing $d=36 \mu \mathrm{m}$. The wires are thin gold on top of $\mathrm{Si}_{x} \mathrm{~N}_{y}$ beams that are $1 \mu \mathrm{m}$ wide. The spacing $d$ is chosen so that it satisfies the equation $d<\lambda / 10$, where $\lambda$ is the incident radiation wavelength. The impedance of the absorber is matched to the incident radiation by tuning the thickness of the Au film on top of the absorber. The incident radiation is polarized and perpendicular to the support beams. (The solid rectangular structures between the support beams are $\mathrm{Si}_{x} \mathrm{~N}_{y}$ and do not affect the functionality of the detector.)

into the beams to pack the detectors into a 2D array for the echelle spectrographs. Because of the illumination pattern of the echelle spectrograph, the spacing between rows can be large and roughly equal to $4 \times \lambda$ where $\lambda$ is the incident radiation. This relatively large space between rows allows us to simply run our dc wiring to the TES between the rows. Bending the beams is straightforward and we have published results that show we can make detectors that can fit into a 2D array [6].

We emphasize that these detectors are mechanically robust due to the very high Young's modulus and yield point of $\mathrm{Si}_{x} \mathrm{~N}_{y}$. Mechanically, one can think of these detectors as a pair of nanowires under stress. Because the mass of the absorbers is so small and the $\mathrm{Si}_{x} \mathrm{~N}_{y}$ is grown so there is $100 \mathrm{MPa}$ of tensile stress, the fundamental resonance $\omega_{\circ}$ is greater than $40 \mathrm{kHz}$ for these detectors. This frequency is much higher than the mechanical vibrations the array would encounter in the field. In fact, this fundamental resonance $\omega_{\circ}$ is close to 10 times higher than JPL's spider-web bolometers which have been flight qualified for Herschel/Planck [13].

Vibrations in the absorbers are not the issue. Instead, the very small thermal conductance $G$ means that the responsivity defined as $\Re \equiv \delta I / \delta P$ is large for our TESs since $\Re \propto G^{-1 / 2}$. Therefore, one must take care to filter the bias lines and shield the array to prevent spurious currents into the circuit. The design of BLISS, however, 
allows one to fully enclose the array in a superconducting box which makes the shielding relatively easy. This is an advantage the BLISS design has over instruments that measure the $\mathrm{CMB}$. The array for an instrument that measures the CMB must open to a large aperture, making electromagnetic shielding more difficult.

\section{MEASUREMENT OF SILICON NITRIDE MESH ABSORBERS}

To determine the heat capacity of the absorbers, we performed noise thermometry measurements on suspended $\mathrm{Si}_{x} \mathrm{~N}_{y}$ absorbers with the geometry shown in Fig. 2. The supports that compose the absorber are $1.0 \mu \mathrm{m}$ wide. The beam dimensions of the supports between the absorber and substrate are $975 \mu \mathrm{m}$ long by $0.4 \mu \mathrm{m}$ wide. We have built absorbers with thicknesses of $0.125 \mu \mathrm{m}$ and 0.25 $\mu \mathrm{m}$. Two $50 \mathrm{~nm}$ thick Au resistors are defined on the absorber. One is used to determine the temperature of the absorber by measuring its Johnson noise and the other is a heater. On some of the absorbers, a $1.0 \mathrm{~nm} / 10 \mathrm{~nm}$ $\mathrm{Ti} / \mathrm{Au}$ metal film is patterned on top of roughly half of the membrane. The total area of the absorber which includes the area underneath the $\mathrm{Au}$ films equals $1.46 \times 10^{4}$ $\mu \mathrm{m}^{2}$. On the bottom side of the $\mathrm{Si}_{x} \mathrm{~N}_{y}$ is a thermal $\mathrm{SiO}_{2}$ layer that is $10 \mathrm{~nm}$ thick. This layer protects the $\mathrm{Si}_{x} \mathrm{~N}_{y}$ from $\mathrm{XeF}_{2}$ gas that we use to surface micromachine the absorber. Finally, superconducting $20 \mathrm{~nm}$ thick Al wires run along the support beams and electrically connect the thermistor and heater to a SQUID amplifier and current source, respectively.

We measure the heat capacity $C$ of the absorber as a function of substrate temperature $T$ by first measuring the response time $\tau$ and the thermal conductivity $G$ as a function of $T$. To measure $\tau$, a small heat pulse is applied to the heater and the change in temperature is measured via the Johnson noise thermometer. (See ref. [8] for further details.) The thermal conductance $G(T)$ is determined by applying power $P$ to the heater, by measuring the temperature $T$ of the membrane, and by using the definition $G(T) \equiv d P / d T$. We determine $C(T)$ using the relationship $C=\tau(T) \times G(T)$.

\section{RESULTS \& DISCUSSION}

Table 2 shows the results for several different samples. The heat capacity $C$ and thermal conductance $G$ were measured from about $30 \mathrm{mK}$ to $600 \mathrm{mK}$. We show the value for $C$ and $G$ when the temperature is $60 \mathrm{mK}$ because this is roughly the lowest temperature that TESs can reach given the current state-of-the-art cryocoolers suitable for space. (The temperature dependence of $C(T)$

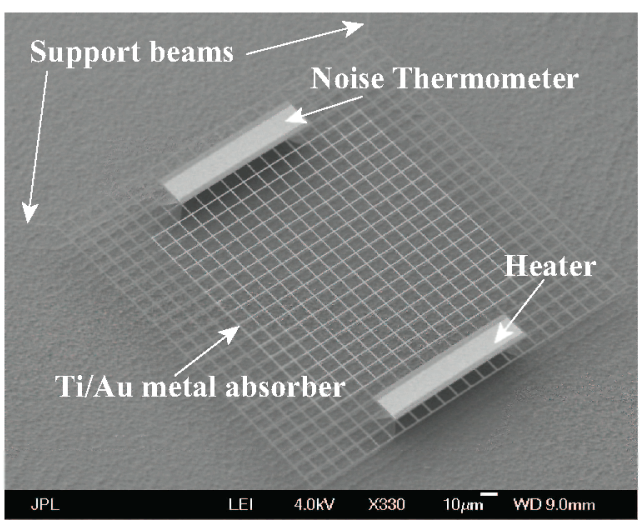

FIGURE 2. Micrograph of suspended mesh $\mathrm{Si}_{x} \mathrm{~N}_{y}$ absorber with a Au thin film heater and Johnson noise thermometer. Support beam dimensions are $975 \mu \mathrm{m}$ long by $0.4 \mu \mathrm{m}$ wide. The $\mathrm{Si}_{x} \mathrm{~N}_{y}$ absorber is $240 \mu \mathrm{m}$ on a side with a $1.0 \mathrm{~nm} / 10 \mathrm{~nm}$ $\mathrm{Ti} / \mathrm{Au}$ metal film patterned on top of roughly half of the absorber. The mesh consists of $1.0 \mu \mathrm{m} \mathrm{Si}{ }_{x} \mathrm{~N}_{y}$ beams with a pitch of $10 \mu \mathrm{m}$. The total area of the absorber which includes the area underneath the thermometer and heater is $1.46 \times 10^{4} \mu \mathrm{m}^{2}$. The pitting underneath the membrane is caused by the dry release process.

and $G(T)$ have been discussed elsewhere [8].) The quantity $N$ is the exponent in the expression $G(T) \propto T^{N}$. The NEP is derived from inserting $G$ from column 2 into the equation for the fundamental temperature fluctuation noise: $N E P \simeq\left(4 k_{b} G T_{c}^{2}\right)^{1 / 2}$ where $k_{\mathrm{b}}$ is Boltzmann's constant, $T$ is the temperature of the absorber, and $G$ is the thermal conductance between the substrate and the absorber [14].

The magnitude of $C$ is much higher than one would expect from the predictions of the Debye model. The volume of the gold on the absorber is about $200 \mu \mathrm{m}^{3}$ so it should contribute only about $1-2 \mathrm{fJ} / \mathrm{K}$ to the heat capacity [15]. The $\mathrm{Si}_{x} \mathrm{~N}_{y}$ is an insulator so according to the Debye model its contribution to $C$ should drop as $T^{3}$ and become negligible at $60 \mathrm{mK}$. Clearly, there are additional microstates on the absorber that are causing an excess heat capacity. One potential source of the excess heat capacity is magnetic impurities in the gold. For Device 1, 2, and 3, the gold that was used to pattern the heater, the Johnson noise thermometer, and the absorber was from the same e-beam evaporator crucible with a nominal purity of $99.99 \%$. To see whether this gold was the cause of the excess $C$, for Devices $4-7$, we used a new crucible with a nominal gold purity of $99.999 \%$. The change did not make a difference. Therefore, we are left to conclude that microstates on the surface or within the bulk of the $\mathrm{Si}_{x} \mathrm{~N}_{y}$ are the dominate source of the heat capacity.

One may ask whether it is possible to determine if the extra microstates lie on the surface or inside the bulk of 
TABLE 2. Measured heat capacity $C$ and thermal conductance $G$ at $60 \mathrm{mK}$ for noise thermometry devices with the geometry shown in Fig. 2. All the devices come from different wafers except Devices 4,6 , and 7 which were diced from the same wafer. Only Devices 1 and 2 have the metal mesh patterned as shown in Fig. 2. The other devices have no metal mesh. Column 3 is the thickness of the $\mathrm{Si}_{x} \mathrm{~N}_{y}$ absorber. The NEP is derived from the equation $N E P=\left(4 k_{b} G T_{c}^{2}\right)^{1 / 2}$ where we assume $G$ from column 2 and $T_{C}=60 \mathrm{mK}$.

\begin{tabular}{cccccc}
\hline Device & $\mathbf{C}(\mathbf{f J} / \mathbf{K})$ & $\mathbf{G}(\mathbf{f W} / \mathbf{K})$ & $\mathbf{S i}_{x} \mathbf{N}_{y}(\mu \mathbf{m})$ & $\mathbf{N}$ & $\mathbf{N E P}\left(\mathbf{W} / \mathbf{H z}^{\mathbf{1} / 2}\right)$ \\
\hline 1 & - & 25 & 0.25 & 0.5 & $7 \times 10^{-20}$ \\
2 & 85 & 40 & 0.125 & 0.5 & $8 \times 10^{-20}$ \\
3 & 130 & 65 & 0.25 & 0.5 & $1 \times 10^{-19}$ \\
4 & 100 & 65 & 0.25 & 1 & $1 \times 10^{-19}$ \\
5 & 90 & 100 & 0.25 & 0.5 & $1.5 \times 10^{-19}$ \\
6 & 80 & 40 & 0.25 & 0.5 & $8 \times 10^{-20}$ \\
7 & 100 & 40 & 0.25 & 0.7 & $8 \times 10^{-20}$ \\
\hline
\end{tabular}

the $\mathrm{Si}_{x} \mathrm{~N}_{y}$. If the excess $C$ is in the bulk, then one might expect Device 2 to have a lower heat capacity since its nitride is half the thickness of the other samples. In fact, Device 2 is close to the average value, although there is sizable scatter in the data. We note that the nitride for all the wafers was grown in a single run. Therefore, impurities inside the nitride should be the same for all the devices leading Device 2 to have the smallest $C$ if the excess heat capacity is in the bulk of the $\mathrm{Si}_{x} \mathrm{~N}_{y}$.

To understand how the $\mathrm{XeF}_{2}$ etch we use to surface micromachine the absorbers might affect $C$ and $G$, we etched Devices 6 and 7 twice as long in the $\mathrm{XeF}_{2}$ etcher as Device 4. (All three devices come from the same wafer.) The longer etch does not seem to change $C$ but the thermal conductance $G$ may be lower for the longer etched devices. However, again the scatter in the data makes it difficult to draw any firm conclusions.

The average heat capacity for these devices is about $100 \mathrm{fW} / \mathrm{K}$. If we define the average surface specific heat $C_{S}$ to equal the average heat capacity divided by the total exposed surface of the absorber, then $C_{S}$ equals about $3 \mathrm{aJ} /\left(\mu \mathrm{m}^{2} \mathrm{~K}\right)$. If we compare this value to other groups [10], we find that it is close to a factor of 10 greater. The reason for this higher surface heat capacity is unclear. Perhaps, our dry surface micromachining process is leaving residue on the surface. We release the absorber using $\mathrm{XeF}_{2}$ gas and we ashe a protective photoresist layer on the top surface of the nitride as the final process step. Could these steps be the cause of the excess heat capacity?

All of the devices with the exception of Devices 4 and 7 show that $G$ follows the power law $G \propto T^{1 / 2}$. This power law is consistent with effective elastic scattering of transport phonons down the beam [16]. Four phonon modes contribute to the thermal transport through our support beams: dilational, torsional, and two longitudinal modes. It is the longitudinal modes' dispersion rela- tion that give rise to the power law $G \propto T^{1 / 2}$ (see [16] for details). As with the heat capacity, the thermal conductance for Device 2 is not smaller than the other devices (within the scatter of the data). This is surprising since the thickness of the support beams is cut in half for this device.

\section{CONCLUSIONS}

Building absorber-coupled TESs for BLISS requires sensitivities less than $10^{-19} \mathrm{~W} / \mathrm{Hz}^{1 / 2}$ and response times below $100 \mathrm{~ms}$. Using a noise thermometry technique, we have found that the heat capacity in our optical absorbers is much higher than one would expect from the Debye model. Our preliminary results suggest that the heat capacity does not scale with the thickness of the nitride membranes and may be dominated by surface states. Nonetheless, by using a mesh absorber geometry, we can build TESs with a response time adequate for BLISS.

\section{ACKNOWLEDGMENTS}

This research was carried out at the Jet Propulsion Laboratory and Caltech under contract with the National Aeronautics and Space Administration.

\section{REFERENCES}

1. C. M. Bradford et al., Proc. SPIE, 6265, 62650U (2006).

2. D. Hughes et al., Nature, 394, 241 (1998).

3. T. Matsumoto, Proc. SPIE, 5487, 1501 (2004).

4. M. Kenyon et al., Proc. SPIE 6275, 627508 (2006).

5. M. Kenyon et al., Nucl. Instrum. Methods Phys. Res. Sect. A 559, 456 (2006).

6. M. Kenyon et al., J. Low Temp. Phys. 151, 116 (2008).

7. K. D. Irwin, $A P L$ 66, 1998 (1995).

8. M. Kenyon et al., accepted to IEEE Trans. Appl. Supercond. (2008).

9. W. A. Phillips, Rep. Prog. Phys. 50, 1657 (1987).

10. K. Rostem et al., Proc. SPIE 7020, 70200L-1 (2008).

11. B. L. Zink and F. Hellman, Solid State Comm. 129, 199 (2004).

12. L. Earle et al., Proc. SPIE. 6275, 627510 (2006).

13. A. D. Turner et al., Appl. Opt. 40, 4921 (2001).

14. J. C. Mather, Appl. Opt. 21 (1982) 1125.

15. C. Kittel, Introduction to solid state physics, New York: Wiley, 1986, ch. 6.

16. B. A. Glavin, Phys. Rev. Lett. 86, 4318 (2001). 